\title{
Análisis multimodal de cápsulas de salud: prevención selectiva del gobierno chileno en pandemia ${ }^{1}$
}

\author{
Multimodal analysis of health capsules: Chilean government selective \\ prevention during the pandemic
}

\author{
Javiera Palacios ${ }^{2}$
}

Resumen

La presente investigación busca identificar los topoi argumentativos multimodales de 16 cápsulas audiovisuales de carácter informativo y preventivo emitidas por el Ministerio de Salud (MINSAL) del Gobierno de Chile de marzo a junio de 2020 en el marco de la pandemia mundial por COVID-19. Con tal propósito, se toma la base teórica de topoi (Wodak, 2003) como articulador argumentativo; los discursos de autoridad como generadores de representaciones mentales; y el rol multimodal para la emisión de mensajes de poder. Estos referentes conceptuales, junto a los postulados de los Estudios Críticos del Discurso (ECD), permiten identificar la función de los topoi y observar los mensajes multimodales dirigidos a la población, tomando como recursos centrales la visualidad (Abril, 2007), el tratamiento textual (Pardo, 2007) y sonoro (van Leeuwen, 1999). Los hallazgos indican que los topoi analizados muestran incongruencias discursivas en los diferentes recursos, donde, a pesar de emitir explícitamente informaciones preventivas, existen discursos implícitos que segmentan la imagen de "país ideal". Bajo esa figura se manifiestan estereotipos que ocultan la diferencia, demostrando dialécticamente que los mensajes de cuidado de parte de la autoridad no velan por todos los chilenos.

Palabras clave: COVID-19, Chile, multimodalidad, topoi, ECD.

\begin{abstract}
This study aims to identify the multimodal argumentative topoi in 16 informational and preventive audiovisual capsules by the Chilean Ministry of Health, from March to July 2020, in the context of the COVID-19 global pandemic. For that purpose, the theoretical basis of topoi (Wodak, 2003) is taken as an argumentative strategy, the discourses of authority employed as generators of mental representations and the multimodal role as negotiating messages of power. These conceptual references in addition to the tenets of Critical Discourse Studies (CDS) allow to identify the function of the topoi and to observe the multimodal messages addressed to the population, taking as well the concepts of visuality (Abril, 2007), textuality (Pardo, 2007) and speech-sound relations (van Leeuwen, 1999). The findings indicate that the topoi analyzed show discursive inconsistencies. Despite explicitly transmitting preventive information, there are

1 Una primera versión de este trabajo fue presentada en el curso "Discriminación y movimientos sociales en las Américas desde los estudios críticos del discurso", Doctorado en Estudios Americanos, USACH.

2 Doctora (c) en Estudios Americanos, USACH. Becaria ANID-PFCHA/ Doctorado Nacional/2019-21191221. Correo: javiera.palacios.r@usach.cl. ORCID ID: https://orcid.org/0000-0001-8121-5633
\end{abstract}

Recibido: 21.04.2021

Aceptado: 10.08.2021 


\section{Javiera Palacios}

implicit discourses that segment the image of the "ideal country". According to this, a big part of the Chilean population becomes invisible due to the stereotypes manifested in the capsules, dialectically demonstrating that the messages of preventive care from the authority do not protect all Chilean citizens.

Keywords: COVID-19, Chile, multimodality, topoi, CDS.

\section{Introducción}

El 3 de marzo del año 2020 se marca como hito la llegada del virus COVID-19 a Chile, con el primer caso reportado en la región del Maule por el Ministerio de Salud (MINSAL, 2020a). Ocho días más tarde la Organización Mundial de la Salud (OMS) declara la propagación de esta nueva cepa de coronavirus como una crisis sanitaria y, con ello, el rótulo de pandemia mundial. A raíz de este reporte, Chile comenzó a abordar medidas preventivas para la población en medio de un escenario que, previo a este hecho, ya era complejo político y socialmente. Y es que meses antes, producto del estallido social chileno (Navarro y Tromben, 2019), la sociedad se encontraba fracturada, especialmente en lo referido a la baja credibilidad del poder ejecutivo y legislativo. No obstante, la emergencia sanitaria implicaría un nuevo eje de interés tanto para la ciudadanía como las instituciones gubernamentales.

El gobierno central, como un modo de controlar una incipiente alza de contagios, comenzó a tomar medidas asociadas a las crisis -sociopolítica ${ }^{3}$ y sanitaria-, instaurando un estado de excepción constitucional, con un toque de queda nacional de 22:00 a 05:00 horas (Chileatiende, 2020), cuarentenas y aforos reducidos, controles sanitarios y la presencia en las calles de las Fuerzas Armadas y del Orden, en resguardo de dichas restricciones (Armada de Chile, 2020).

Fue bajo estas condiciones que el Ministerio de Salud dio paso a una campaña comunicacional de carácter audiovisual apuntada a la prevención y cuidados para combatir la propagación del virus y, a su vez, acallar la voz crítica de la ciudadanía y la comunidad médica y científica, quienes solicitaban acciones urgentes para manejar este escenario (Comed, 2020). La estrategia de prevención fue presentada a la comunidad por medio de cápsulas informativas, las que comenzaron a exhibirse por todas las plataformas de difusión gubernamentales, instituciones de salud públicas/privadas, y medios de comunicación nacionales, con el fin de viralizar, paradójicamente, las medidas de cuidado.

De marzo a junio del año 2020, se realizaron más de una docena de cápsulas, las cuales tomaron animaciones, infografías e imágenes de personas anónimas, para promover acciones de resguardo sanitario e informaciones ligadas a la prevención (El Mostrador, 2020a). Sin embargo, en ellas se puede visualizar, a priori, un tipo de canon visual que responde mayoritariamente a la presencia de gente blanca y delgada, sin rasgos indígenas ni origen inmigrante, y cuya situación socioeconómica -vista en los espacios generales donde se desarrolla la campaña- les permitiría

\footnotetext{
${ }^{3}$ Desde el 18 de octubre de 2019 al 13 de marzo de 2020 se realizaron todos los viernes manifestaciones masivas en todo el territorio que paralizaban al país, en las que se demandaba por la desigualdad e inequidad social, impactando a todas las esferas e instituciones (Domingo, 2020).
} 


\section{Javiera Palacios}

enfrentar la pandemia sin verse afectados por factores asociados a la pobreza y hacinamiento. Es por lo mencionado que se tomó esta campaña para ser trabajada bajo los estudios del discurso, con el objetivo de observar estos escenarios en profundidad y conocer las líneas de exposición discursivas desde los diferentes recursos semióticos ${ }^{4}$.

Por tanto, la presente investigación tiene como objetivo identificar los tópicos argumentativos en la multimodalidad de las cápsulas preventivas en el contexto del COVID-19 y observar la función que cumplen con respecto a la exclusión visual de la diferencia apuntada particularmente a este caso, en un país en crisis social y sanitaria. Para ello, se consideraron tres líneas teóricas que toman como base los topoi, entendidos como justificaciones relacionadas con lo argumentativo, o lugares comunes donde se vinculan los mensajes con conclusiones que no son discutibles (Wodak, 2003). A partir de este concepto, se propondrá un giro teórico desde la multimodalidad y el juego entre los diferentes recursos comunicativos (Kress y van Leeuwen, 1996), erigiendo la visualidad como centro y sus líneas estratégicas con lo escrito y lo sonoro. Es desde esa perspectiva que se incluirán las líneas de los Estudios Críticos del Discurso (van Dijk, 1999; Fairclough y Fairclough, 2012; Wodak y Meyer, 2015), donde se abordarán conceptos de segmentación y exclusión de la diferencia.

Las preguntas que orientan este estudio son: ¿cuáles son los topoi presentes y representativos en las cápsulas preventivas? ¿De qué forma los topoi se logran cosificar ideológicamente en los modelos mentales de la audiencia?

El corpus de análisis lo componen las cápsulas preventivas del COVID-19 dirigidas a la población en general por el MINSAL a través de su canal oficial de Youtube durante los periodos de marzo a junio, y que hayan incluido personas. Estos cortos informativos serán analizados desde una óptica investigativa mixta, la que tomará aspectos cuantitativos en torno a frecuencias y usos; y cualitativos en relación a los trabajos de Abril (2007), Pardo (2007) y van Leeuwen (1999) para comprender su materialidad y recursos semióticos visuales, escritos y sonoros. Las cápsulas se abordan desde una perspectiva metodológica que expone, en una primera etapa, los argumentos discursivos en los recursos semióticos, categorizando lo nombrado y lo omitido por medio del análisis multimodal con matices críticos (van Dijk, 1999) y, en segundo lugar, los recursos recogidos se interpretarán y clasificarán por medio de los topoi escogidos para esta investigación. Posterior a esto, se expondrán los puntos metodológicos multimodales abordados y los resultados de análisis de los topoi, para finalizar con las conclusiones del estudio y un llamado a la necesidad de realizar estudios de los discursos y mensajes de autoridad.

\footnotetext{
${ }^{4}$ Para evitar confusiones terminológicas, en este artículo se utilizará el concepto de "recurso semiótico", haciendo referencia a los distintos recursos/modos/soportes de comunicación y de representación que se utilizan para crear significados, ya sean escritos, visuales, sonoros y kinestésicos.
} 


\section{Informaciones de autoridad}

Cuando los mensajes están generados desde instituciones que tienen incidencia en la población, se habla de información de poder, la cual busca tener efectos significativos en las dinámicas del contexto social (Castells, 2009). Esta información está construida materialmente para definir líneas de acción, producir cambios o mantener prácticas que favorezcan el actuar de quienes la emiten (Fairclough, 2003). Asimismo, expone -o reserva- temas, con el fin de construir identidades (van Dijk, 1998). Estas enunciaciones no están dadas de forma aislada, sino que están entretejidas en redes ideológicas articuladas por quien emite el mensaje, por tanto, "nunca un discurso existe por sí mismo sin estar anclado en algún otro" (Íñiguez, 2006, p. 113).

Las relaciones enunciativas y los contenidos de poder pueden visualizarse en dos niveles de interpretación: lo intertextual, referente a la utilización de ideas y temáticas explícitamente presentes en los discursos y, lo interdiscursivo, donde los mensajes están construidos en base a diferentes recursos semióticos que incluyen en su contenido convenciones sociales determinadas (Luzón, 1997). En este contexto, los mensajes de autoridad centran su importancia en el lenguaje, su uso y contenido, los cuales se consideran agentes centrales en los procesos de construcción social de la realidad (Ghio, Navarro y Lukin, 2017).

En esta investigación se toman las cápsulas informativas como un segmento informativo multimodal emitido por el gobierno y que entrega mensajes explícitos y sutiles, con el propósito de controlar las acciones de quienes las reciben (Van Dijk, 1999), pues, independiente de la temática a tratar, el discurso de autoridad se remite a una consigna: "comunicar en política es hacer política" (Navarro y Tromben, 2019, p. 298). Desde esta perspectiva, se busca comunicar de todas las formas discursivas posibles, siendo el formato audiovisual uno de los más multisemióticos dado que posee diversos niveles de producción de significados, observados en lo escrito, visual, sonoro y, en conjunto, cumplen con los requerimientos comunicacionales de las autoridades (Kress y van Leeuwen, 1996). Como una acción de reforzamiento, van Dijk (2012) expone que es vital construir, usar e interpretar los mensajes desde una perspectiva sociocognitiva, la cual relaciona las informaciones dadas a tres dimensiones claves: discurso, cognición y sociedad.

Esta tríada elemental, abordada en la comprensión de los mensajes multimodales de las cápsulas, debe ampliar la comprensión de las representaciones expuestas en los fragmentos que "educan" a la población. De esta manera, el "discurso" alberga las prácticas que toman como base la "sociedad" como el medio en que se despliegan las informaciones institucionales y de cuidado; mientras que la "cognición" refiere a la dimensión individual y social que remite a sus representaciones mentales y contextuales (van Dijk, 2012). Estas dimensiones son esenciales para comprender las informaciones de autoridad; sin ellas no se podrían interpretar las situaciones y estructuras sociales (van Dijk, 1997) ni tampoco establecer relaciones de poder ideológico (Fairclough, 2003).

Para hablar de discurso e ideología, hay que tomar en cuenta que estos conceptos están interrelacionados, pues según los postulados de van Dijk (2003) ambos "influyen en lo que 


\section{Javiera Palacios}

decimos y cómo lo decimos" (p.79), donde destaca el matiz sociocognitivo en la definición de las temáticas y marcos interpretativos que circunscriben el contexto, identidades sociales, posiciones ideológicas, y también, el contenido semiótico que circula en la sociedad, encargado de la producción de las informaciones y mensajes en vínculo con los diversos recursos semióticos multimodales relacionados a las ideas de poder.

Por tanto, se puede exponer que las ideologías no son innatas, sino aprendidas ya que todos los mensajes que circulan, social y contextualmente, se remiten y manifiestan como modelos mentales, los que se traducen en aprendizajes forzosos, ejercidos por parte de los grupos de autoridad (van Dijk, 2012), los que dirigen y proponen, por medio de una serie de categorías discursivas, las líneas de pensamiento de la audiencia a una cierta visión de mundo (Ruiz y Estrevel, 2008).

\section{Argumentos multimodales categorizados en topoi}

Después de visualizar la importancia del soporte, los recursos semióticos y la mediación ideológica de los discursos de autoridad, es de suma relevancia develar los significados y categorizaciones de estos discursos, en este caso particular, describir a qué hacemos referencia con el término topoi. Este concepto, trabajado por Wodak (2003) en los estudios del discurso de la historia, se presenta como una aproximación para comprender los fenómenos sociales, la discriminación y el poder, partes de un imaginario cultural construido y compartido.

En este estudio, los topoi no se tomarán desde la perspectiva aristotélica y de Cirón (Žagar, 2010), sino desde la estructura base de la teoría de la argumentación de Anscombre y Ducrot (1988) abordada por Wodak (2003). En esta línea, el término en cuestión hace referencia a lo que se conoce como "lugar común" en la argumentación, visto como el conjunto de elementos que forman parte de las premisas de autoridad, sean dadas de forma explícita o implícita. Por tanto, los topoi procuran explicar las tensiones que ocurren en la sociedad, pues son utilizados para justificar inclusiones o exclusiones, con el propósito de ordenar -y conectar- los contenidos. Esta transición, desde argumentos a conclusiones (Kienpointner, 1992, en Wodak, 2003), se ejerce con base en depósitos de ideas claves generalizadas (Richardson, 2004) que son expuestas para justificar y aglutinar mensajes simbólicos que generan declaraciones determinadas y logran objetivos propios (Wodak, 2009). En concreto, se trata de estrategias de argumentación conclusivas para la persuasión de significados específicos en una determinada audiencia (Wodak, 2003), las cuales servirían a los grupos dominantes/autoridades para imponer su propia agenda y validar sus discursos ideológicos.

A pesar de que los topoi han sido aplicados principalmente en el estudio del discurso escrito, también pueden ser abordados desde los diferentes recursos semióticos ya que el funcionamiento del topoi no distingue el formato material o estrategia discursiva utilizada (Wodak, 2009). 


\section{Javiera Palacios}

Wodak (2003) distingue diversos tipos de topoi que se manifiestan de manera frecuente en los discursos de poder. Se trata de 15 categorías o estrategias, de las cuales, para este estudio, se seleccionaron cuatro: utilidad, inutilidad, definición (o interpretación de los nombres) y justicia; vistas en la Tabla 1.

Tabla 1: Topoi considerados para esta investigación, con base en Wodak (2003).

\begin{tabular}{|ll|}
\hline TOPOI & DESCRIPCIÓN \\
\hline Utilidad & $\begin{array}{l}\text { Acción que resulta ventajosa o útil desde un determinado punto de vista. Por } \\
\text { tanto, hay que realizarla. }\end{array}$ \\
\hline Inutilidad & $\begin{array}{l}\text { Hechos que resultan inútiles o generan desventajas. Entonces, no hay que } \\
\text { efectuarlas y se deben tratar de evitar. }\end{array}$ \\
\hline Definición & $\begin{array}{l}\text { Si una persona, o grupo de personas, cosa o situación recibe un nombre o } \\
\text { rotulación específica, esta o estos deben responder a esas cualidades, rasgos o } \\
\text { atributos asignados a la etiqueta. }\end{array}$ \\
\hline Justicia & $\begin{array}{l}\text { Situaciones en las que todas las personas comparten aspectos específicos y, por } \\
\text { tanto, deben tener un mismo trato o consideración. }\end{array}$ \\
\hline
\end{tabular}

La selección de estas cuatro categorías toma como guía el criterio de Wodak (2009) para el estudio de los discursos políticos y grupos de poder. Estos topoi son empleados estratégicamente como ejes temáticos para la negociación, justificación y/o cimentación de mensajes en la audiencia, ya que permiten relacionar el conocimiento social-contextual compartido (van Dijk, 2005a) con los mensajes construidos, los que, en su conjunto, cumplen el "propósito de hacer una inferencia particular" (Wodak, 2011, p. 173).

\section{La utilidad de los estudios críticos de los discursos}

Para poder identificar los topoi y su operación dentro de los mensajes multimodales, se hace necesaria la utilización de los estudios críticos del discurso (ECD). Mediante esta herramienta es posible conocer el uso que los emisores le dan al mensaje y a los contenidos discursivos, más aún si son dados por los grupos de poder. Esto se sustenta en la premisa de que "quienes controlan el discurso público controlan ampliamente la mentalidad social e indirectamente la acción pública; y, por consiguiente, controlan también la estructura social" (van Dijk, 1999, p. 34).

Por lo anterior, resulta de interés estudiar los discursos desde una perspectiva crítica, ya que este concepto se centra en conocer los efectos de las situaciones sociales-discursivas de poder y el uso dado en los contextos particulares, especialmente en lo relacionado con la reproducción multimodal de ideologías entendidas como representaciones (van Dijk, 1999). Dichas representaciones pueden ser vistas desde la multimodalidad, perspectiva que analiza las funciones 


\section{Javiera Palacios}

de los signos y los sistemas de significación según cada recurso semiótico, por separado y en su conjunto, permitiendo una conexión analítica con otros modos/recursos de forma compleja (Kress y van Leeuwen, 1996).

La negación a la diferencia es un tema recurrente en los ECD, y es en los discursos multimodales de autoridad donde más se puede ver la polarización del nosotros y ellos (van Dijk, 1999), algunas veces de forma directa y en otras de manera sutil, remitiéndose en ambos casos, de forma predominante, a discursos o representaciones mentales anteriores (Wodak, 2015). Decir nosotros es referir al grupo con el cual los productores del discurso se sienten identificados, y donde comparten e interpretan de forma símil la realidad. En contraste, Ellos -o los otrosrepresentan un "peligro" o algo alejado de la concepción de nosotros, o lo que estos consideran correcto o necesario (Sáez, 2014). El enfrentamiento de ambos grupos muchas veces está constituido por características o rotulaciones (van Dijk, 2005b), las que se visibilizan cuando son favorables, o disimulan cuando están en contra. Este es un modo de mostrar la superioridad sobre el otro y de valorar sus apariciones en desmedro de los ocultados o negados; todo ello con fines de persuasión (van Dijk, 1998).

De esta forma, el visualizar los discursos de autoridad por medio de los ECD es una manera de exponer las rutinas segmentadoras dominantes, pero no desde la búsqueda por develar las mediaciones presentes, sino para comprender cómo se manifiestan en una campaña de prevención de salud.

\section{Metodología}

Como técnica de recolección, se seleccionó un corpus de análisis compuesto por cápsulas informativas audiovisuales, centradas en informaciones temáticas específicas sobre el cuidado del coronavirus, y que fuesen emitidas por la página oficial del MINSAL en la plataforma Youtube. En este marco, se consideró como periodo de inicio marzo de 2020, marcado por el primer caso de contagio en el país, y junio del mismo año, momento en que comienza a bajar la curva de contagio (MINSAL, 2020b). Siguiendo un criterio característico de uniformidad y contenido, se excluyeron los videos que contenían sólo infografías o animaciones, dejando únicamente seleccionados los que contaran con presencia de personas. Conforme a esta selección, fueron 16 las cápsulas informativas analizadas.

La técnica de análisis implicó el desarrollo de una metodología específica para efectos de esta investigación, la cual responde a una perspectiva de carácter mixto, con usos cuantitativos y cualitativos. Para ello, se tomaron como base los postulados teóricos de Wodak (2003) sobre los tópicos de autoridad, y de van Dijk $(1999,2012)$ en torno a los modelos mentales y discursos de poder. Este modo de trabajo considera, también, los postulados de Fairclough y Fairclough (2012) en relación a los estudios del discurso político; algunas perspectivas de Wodak y Meyer (2015) respecto a los ECD; y O'Halloran (2012) sobre los tratamientos discursivos multimodales audiovisuales. 


\section{Javiera Palacios}

En materia operacional, los aspectos señalados referentes al estudio del discurso se establecen como ejes transversales de análisis en las cápsulas informativas, en las que se toma el contenido multimodal como base. En él se comprende que todos los recursos semióticos de comunicación -y la interrelación de ellos- se configuran como unidades de significados complementarias entre sí. Por tanto, la perspectiva multimodal contribuye a la creación de sentido de las audiencias receptoras, las que influyen en los estímulos sensoriales de manera directa y, a su vez, potencian la interpretación.

En cuanto al tratamiento multimodal, la gramática visual de Kress y van Leeuwen (1996) y la perspectiva de O'Halloran (2012) se complementan en la observación de cada recurso bajo sus propias características y lecturas. Desde ambas premisas, es posible realizar un análisis combinatorio de intersemiosis sobre cada enunciación, las cuales, a pesar de provenir de diferentes naturalezas semióticas, coexisten y representan un discurso particular, contenedor de mensajes socialmente construidos. En este estudio, la construcción de estos mensajes está también fuertemente relacionada con las perspectivas ideológicas y el componente contextual propuestos por van Dijk (2003).

De acuerdo a los aportes mencionados, la metodología busca identificar los topoi discursivos multimodales en los modos semióticos de comunicación involucrados en esta investigación: visual, escrito y sonoro, tanto por separado como todos en su conjunto, utilizando una observación multimodal de dos pasos. En una primera etapa (Figura 1) se visualizan e identifican los contenidos expuestos en las cápsulas de salud por recurso semiótico en particular y las posteriores interacciones entre ellos. En este punto, se subdividen los modos discursivos en tres micro categorías de análisis, los cuales incluyen la presencia de planos y marcos visuales de los recursos asociados a la imagen como cerco de identificación de elementos y situaciones de carácter evidente (Abril, 2007). En lo sonoro, se aborda la música de apoyo y voces enunciadoras que soportan los principales aspectos comunicativos (van Leeuwen, 1999) y, por último, los subtítulos, textos y modos escritos que apoyan y sostienen los mensajes (Pardo, 2007) dados en las cápsulas informativas. 


\section{Javiera Palacios}

Figura 1: Ejemplo de método de análisis multimodal, aplicado al caso.

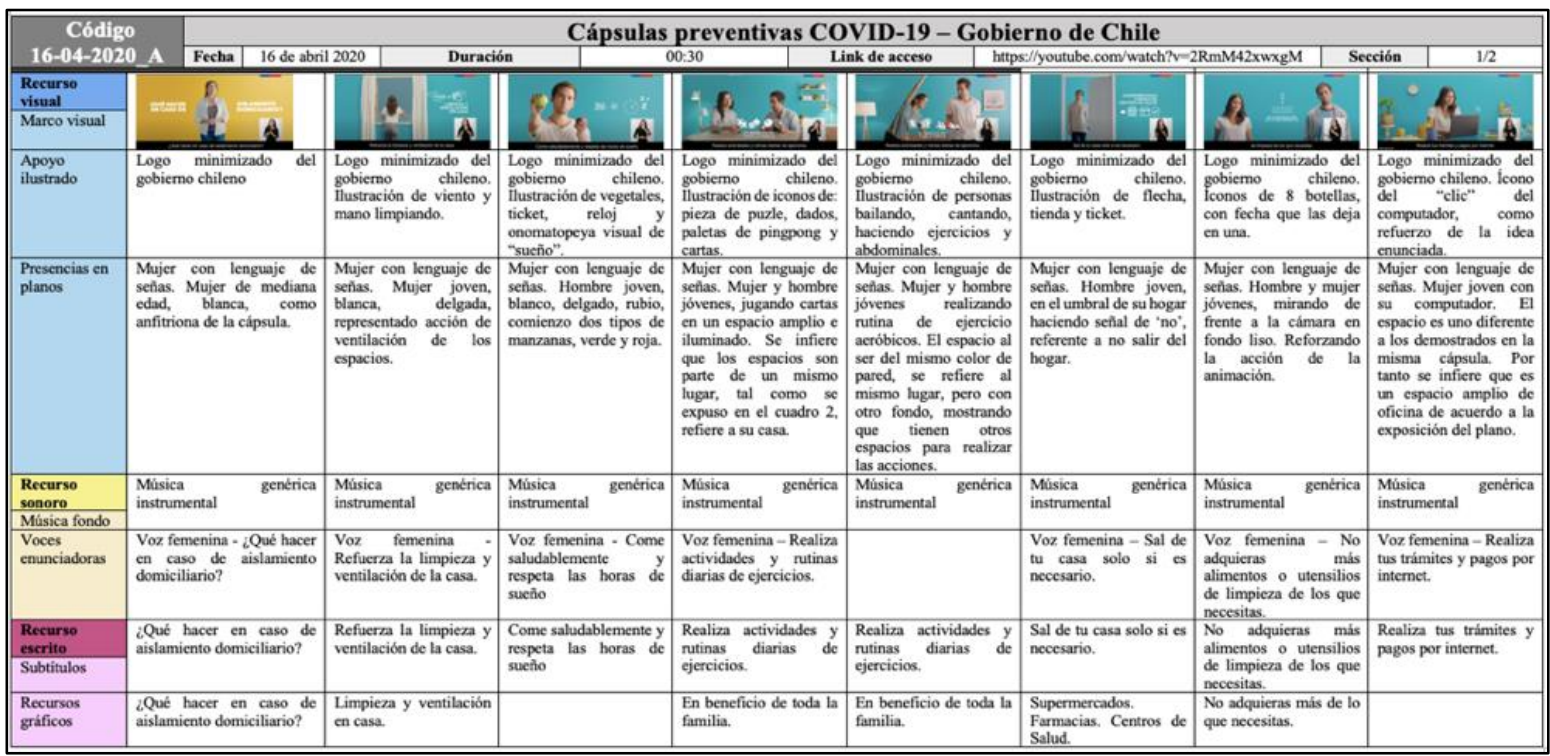

Con el material recogido en bruto -según las microcategorías evidenciadas- se procedió en una segunda etapa a categorizar de modo conceptual los cuatro topoi seleccionados para esta investigación, siguiendo a Wodak (2003): utilidad, inutilidad, definición y justicia. Cada uno se cuantificó según las similitudes tópico-discursivas; primero, desde una lógica de frecuencia/aparición temática por recurso semiótico y, posteriormente, desde un sentido cualitativo, permitiendo con ello, interpretar los datos arrojados de forma explícita e implícita y, en paralelo, evidenciar las ausencias. Después de esta división se ordenaron los ítems según subcategorías argumentales, las cuales respondieron a la asociación por medio de interpretaciones temáticas de los estímulos semióticos, con el fin de realizar una observación crítica sobre los mensajes multimodales dados por el gobierno chileno en el marco de la temática global del COVID-19.

\section{Análisis, resultados y discusión}

En el análisis se abordaron 16 cápsulas informativas de manera individual. Luego se realizó una cuantificación general y según cada recurso semiótico expuesto en fotogramas de ejemplos (Figuras 4-8). Los videos fueron $\operatorname{codificados}^{5}$ para identificarlos según el topoi y recurso correspondiente y, así, visualizar los resultados de investigación situados bajo la consigna general del cuidado de la población por parte del MINSAL. Vale la pena recalcar que al tratarse de información generada por el gobierno, constituye un discurso de autoridad; por tanto, prevalece la

\footnotetext{
${ }^{5}$ La codificación de cada cápsula responde a la siguiente identificación: fecha de publicación y al orden de subida al canal oficial de Youtube de la entidad. Esta organización se produce debido a que, en la mayoría de las veces, se estrenó más de un video por día. Por tanto, se obtiene un código del tipo: 18-03-2020_A
} 


\section{Javiera Palacios}

intención de alinear el actuar de sus ciudadanos a partir de lo que se considera correcto o beneficioso para el grupo dominante (Wodak, 2003) y, junto a esto, justificar sus imaginarios bajo una rutina multimodal-contextual que no es desconocida para la audiencia. Con esto, se ayuda a reforzar los modelos mentales y estereotipos de la población.

\section{Identificación general de los topoi}

Desde una mirada global de las cápsulas estudiadas, se puede decir que un $100 \%$ de los videos preventivos contó con alusiones temáticas relacionadas a los cuatro topoi propuestos -utilidad, inutilidad, definición y justicia. Asimismo, los videos contaron con subcategorías de presencia y uso por cada recurso, las cuales no fueron necesariamente similares de acuerdo a su argumentación. De acuerdo a esto último, el estudio de lo visual, lo escrito y lo sonoro contó con 15 subcategorías por cada ítem, de las cuales siete fueron compartidas entre los tres recursos semióticos. También se presentaron subcategorías duales -compartidas entre dos recursos- e individuales de cada sistema discursivo, como se muestra en la Figura 2.

Figura 2: División de subcategorías por recurso.

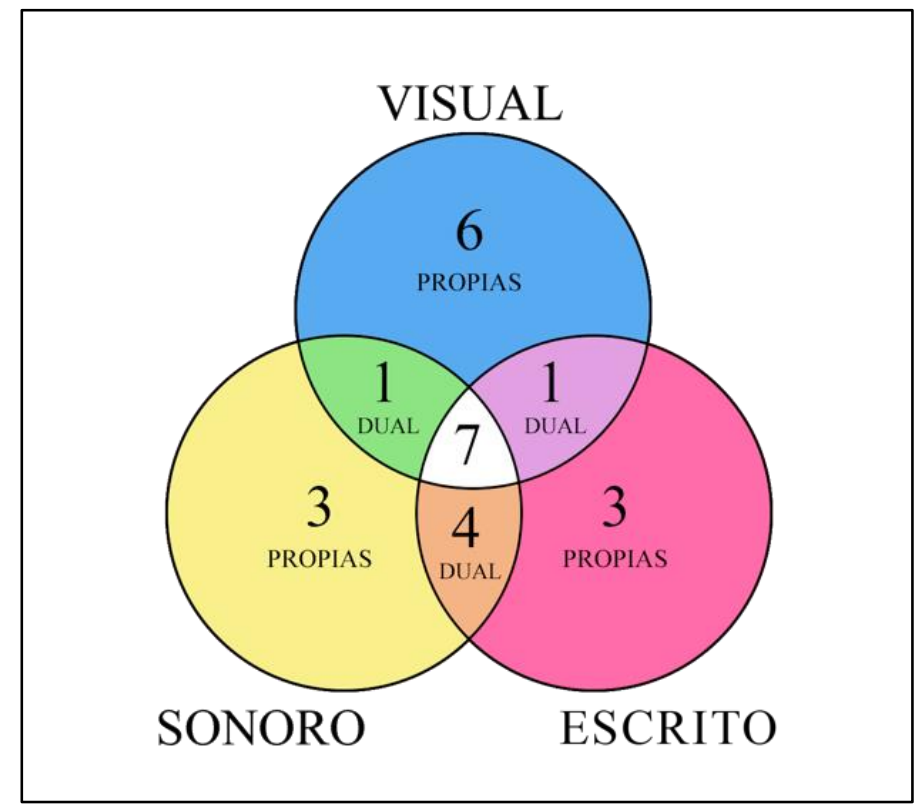

Las siete subcategorías compartidas por los tres recursos discursivos se agruparon de acuerdo a los cuatro topoi considerados para esta investigación, permitiendo, de esta manera, visualizar la frecuencia de aparición y conocer los argumentos temáticos predominantes en las cápsulas preventivas analizadas (Figura 3). Desde esa perspectiva, el topoi más ocupado es utilidad $(57,9 \%)$, referente a las acciones que los ciudadanos deben realizar, como al cuidado preventivo frente al virus y otras informaciones asociadas a lo que el grupo de poder quiere que la población 


\section{Javiera Palacios}

realice. Por otra parte, el nivel menos usado es el de inutilidad (7,5\%), basado en restricciones preventivas relacionadas a las acciones que, de acuerdo al contexto de pandemia, no hay que hacer.

Figura 3: Utilización por topoi, según subcategorías compartidas.

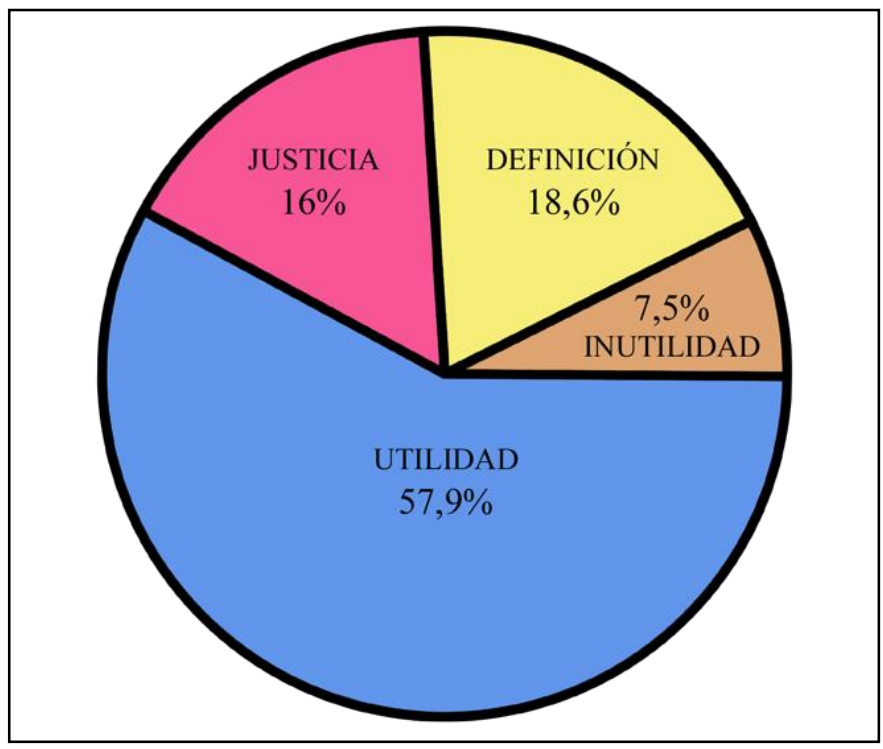

Para comprender las sutilezas de cada sistema discursivo y sus respectivas estrategias argumentales, se generaron subcategorías desglosadas por topoi. Por la extensión de los resultados, se abordarán sólo los dos tópicos argumentales más utilizados en las cápsulas preventivas dadas por el MINSAL.

\section{a. Topoi utilidad}

Con un uso compartido entre lo escrito, visual y sonoro, la subcategoría procedimiento fue la más utilizada a nivel de frecuencia. Al tratarse de una cápsula de cuidados de la salud, la observación se centró informacionalmente en explicitar acciones a concretarse bajo el discurso del hacer, como se aprecia en la Figura 4, mediante la acción de lavarse las manos con agua y jabón por al menos 30 segundos, algo que es reafirmado por los tres recursos semióticos de forma simultánea con una aparición de 108 veces. Si se analizan discursivamente, estas acciones se constituyen en "lugares/conocimientos comunes" debido a que por medio del reforzamiento de los procedimientos preventivos en los diferentes recursos, remiten a la audiencia a informaciones/modelos mentales anteriores dados como verdaderos, donde emisores y receptores comparten las creencias emitidas (Anscombre y Ducrot, 1983). Al igual que los mensajes, estos "lugares comunes" permiten validar a quienes dan los mensajes, estableciéndose como una fuente enunciativa creíble (Pardo, 2007). Por tanto, el gobierno chileno, además de dar mensajes ligados a la prevención de salud, busca reforzar su rol y voz de autoridad en momentos de crisis. 


\section{Javiera Palacios}

Figura 4: Subcategoría procedimiento (18-03-2020_B).

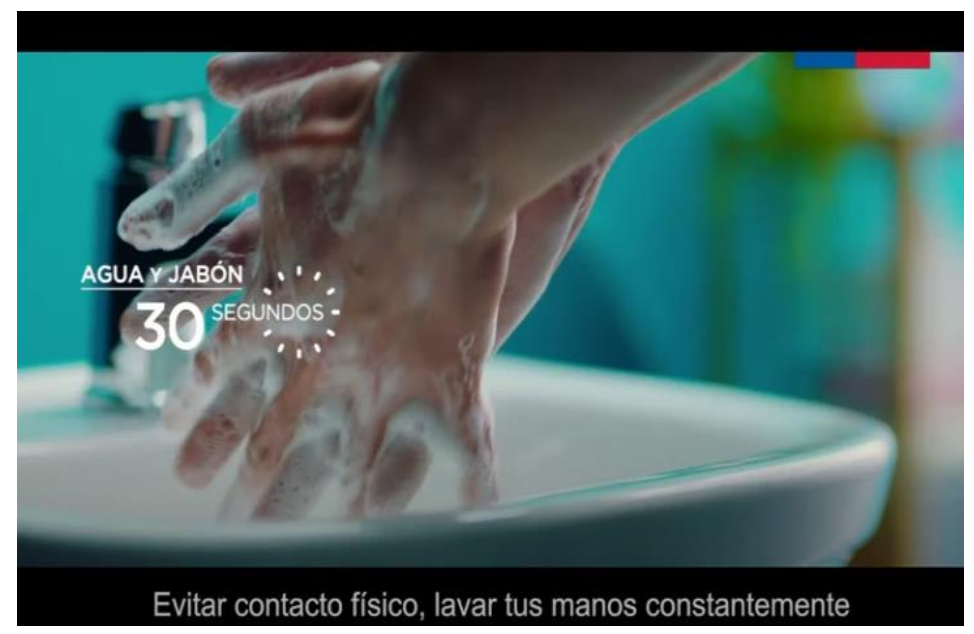

Fuente: MINSAL.

Otra acción concreta que fomentan las cápsulas es la subcategoría de la conectividad, la cual está presente en todos los recursos semióticos analizados. Este recurso retrata a los protagonistas de los videos usando computadores (Figura 5), tablets y teléfonos inteligentes con diversos fines útiles, como contactar al número de teléfono o página web del MINSAL, o realizar actividades online bajo confinamiento. Todo esto es reforzado visualmente en 25 oportunidades, y 20 veces de forma oral y escrita.

En el análisis de estos recursos informativos se pudo constatar que, a pesar del fomento de este tipo de actividades, según las investigaciones realizadas por la Subsecretaría de Telecomunicaciones (2018), el acceso a internet es dispar en el país: sólo un 27,2\% de los chilenos cuenta con internet banda ancha e internet móvil, un $29 \%$ con acceso fijo y un $28,9 \%$ con acceso móvil únicamente. A esto se suma que no todos los hogares cuentan con equipos computacionales o inteligentes para tener acceso a la red ni el espacio físico cómodo, amplio e iluminado, como el que se muestra en la Figura 5. De esta manera, los mensajes asociados a recomendaciones para el cuidado personal, conocer dónde y cómo ir a los centros asistenciales, o realizarse un test preventivo, son informaciones a las que sólo algunos, aquellos que tengan las herramientas tecnológicas referidas, pueden acceder. 


\section{Javiera Palacios}

Figura 5: Subcategoría conectividad (16-04-2020_A).

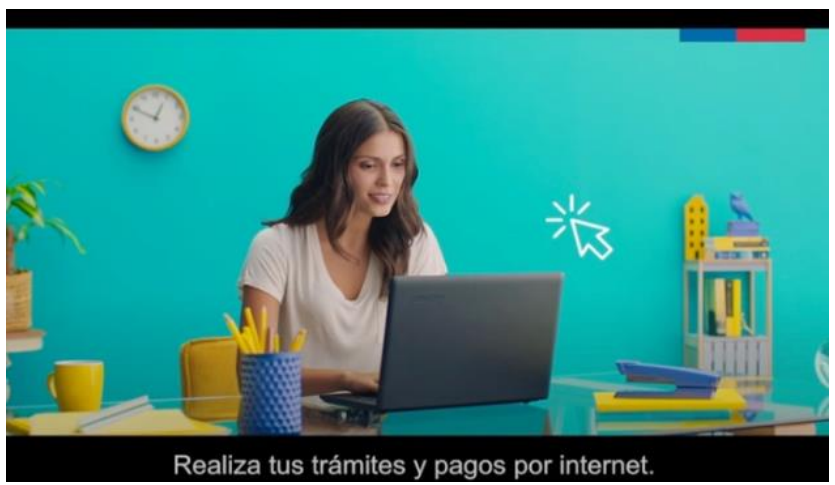

Fuente: MINSAL.

\section{b. Topoi inutilidad}

En las cápsulas preventivas sólo fue encontrada una subcategoría: restricciones preventivas (Figura 6), manifestada en los tres recursos estudiados. Cabe destacar que este fue uno de los mensajes menos utilizados durante la campaña contra el COVID-19, propiciado por un plan inicial del MINSAL enfocado en "lo que hay que hacer" en desmedro de lo que "no se debe hacer", o que pueda generar amenaza (repetición de NO y SIN, en Figura 6). Se infiere que esta estrategia responde a que las personas aceptan y reciben mejor las informaciones positivas que aquellos mensajes negativos que restringen el actuar. En concreto, el decir “qué hacer” está directamente relacionado con cómo se enuncian los mensajes, los nexos mentales y las representaciones contextuales, constituyendo un conjunto de elementos que influyen directamente en el decir y actuar (van Dijk, 2003).

Figura 6: Subcategoría restricciones preventivas (28-05-2020_A).

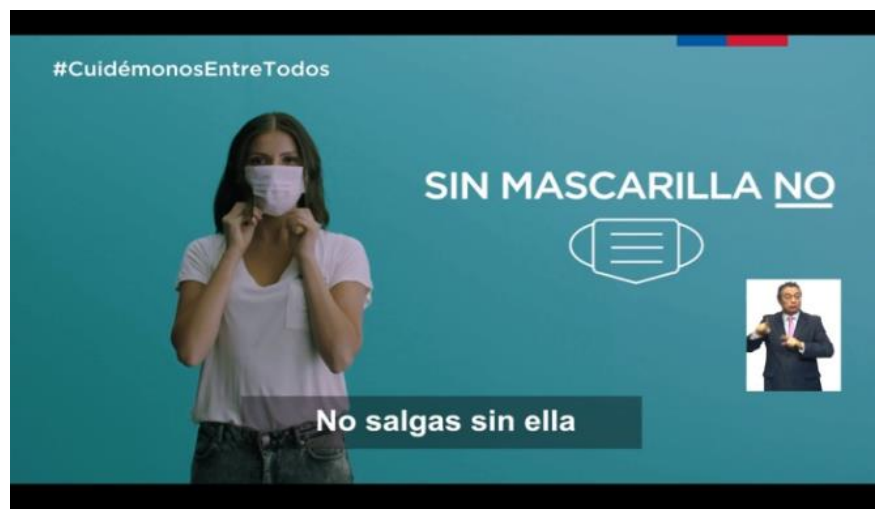

Fuente: MINSAL. 


\section{Javiera Palacios}

Paralelamente, se destaca que las prevenciones explicitadas en el video remiten a un contexto social chileno incongruente con la emergencia sanitaria. Al respecto, se consignan mensajes como "iQuédate en casa y respeta la cuarentena!" (18-03-2020_B), o "Evita ir a lugares donde no puedas mantener un metro de distancia con otra persona" (28-03-2020_B); recomendaciones que, tomando en cuenta la fecha de emisión de ambas cápsulas, responden a un periodo en que aún no se decretaba cuarentena obligatoria y, por tanto, no se condecían con la realidad del transporte público y el importante número de personas que hace uso de éste para desplazarse a sus lugares de trabajo y hogares (La Tercera, 2020a).

\section{c. Topoi definición}

Siguiendo el tratamiento de Wodak (2003) para este nivel de topoi, cuando una acción, cosa o grupo se rotula bajo un nombre, ésta debe actuar, tener rasgos o atributos literales de ese calificativo. Es bajo esta perspectiva que el Gobierno de Chile y el MINSAL ocupan su propia figura, expuesta en la subcategoría autorepresentación. Esta estrategia es visible en 183 menciones multimodales presentes bajo la figura de protección a la comunidad y la autoproclamación de guía en tiempos de crisis. Así, desde una representación de autoridad para la audiencia, se releva la visión del nosotros sobre ellos. Esto es observable cuando se hace un repetitivo llamado a contactar las redes de apoyo, denunciar y/o informarse sólo por el contenido expuesto en los medios del gobierno, haciendo un llamado constante de "infórmate en fuentes oficiales" (04-03-2020_A; 2803-2020_A; 16-04_2020_A) por los tres recursos semióticos de forma individual o en su conjunto. Analíticamente, este punto es de suma importancia, ya que estas cápsulas fueron emitidas en el contexto en que el Ministerio de Salud y el propio gobierno estaban siendo cuestionados por la ciudadanía y el COMED (La Tercera, 2020b). De esta manera, la autoridad y su uso de la autorepresentación hacen un guiño contextual multirecurso, en que la prevención y hacer las cosas correctamente está alineada bajo la figura de lo que nosotros entregamos frente a lo que otros grupos informan.

Paralelamente, la estrategia de autoridad busca una forma simbólica de dar el ejemplo de las acciones que se deben hacer para la prevención. En este punto se exponen 10 apariciones con el logo de gobierno, el cual muestra y establece una 'distancia social'. En esta subcategoría de autorepresentación, a diferencia de otras, el recurso sonoro cumple un rol fundamental e inmediato, pues la música distintiva de las informaciones del gobierno y el reforzamiento representacional e intencional- de la institucionalidad hacen que la audiencia se involucre y conecte con un sonido que conoce y del que se siente parte (van Leeuwen, 1999).

En el recurso visual, vale destacar tres subcategorías ideológicas que emergen desde lo explícito, pero que tienen raíces profundas: representatividad, canon racial y canon físico (Figura 7). En el marco de las intenciones de hacer una cápsula preventiva transversal a la población, se muestran en los videos a hombres, mujeres, una niña y un anciano (61 apariciones), con el propósito de transmitir diversidad etaria y validar las cualidades de un gobierno inclusivo. Sin 


\section{Javiera Palacios}

embargo, las personas elegidas en el casting responden a estereotipos definidos. El primero de ellos responde a un canon racial -127 apariciones- y alude a personas mayoritariamente blancas o medianamente morenas, omitiendo la diferencia y diversidad racial en relación a personas que se consideran parte de una etnia indígena -compuesta por un 12,8\% de la población (INE, 2017)-, o miembros de la población inmigrante -la cual asciende a un número de 1.492.522 residentes (INE, 2019), entre los cuales una mayoría son afrodescendientes. Por otra parte, a nivel físico, se muestra a personas delgadas y aparentemente sin "imperfecciones" -134 apariciones-, omitiendo cánones físicos disímiles, rasgos extravagantes y personas en condición de discapacidad. De esta manera, se considera sólo a quien cumpla con los estándares saludables, apuntando el mensaje a un tipo específico de personas que cumple con los lineamientos que la autoridad considera parte del nosotros. Se evoca, así, la conformación del tejido social de acuerdo a una "realidad" visual (Abril, 2007) que es justificada y forjada en base a conclusiones sociales (Taboada, 2018), por medio de un conocimiento compartido previamente y aceptado culturalmente (van Dijk, 2005a).

Figura 7: Subcategoría representatividad, canon racial y canon físico (28-05-2020_A).

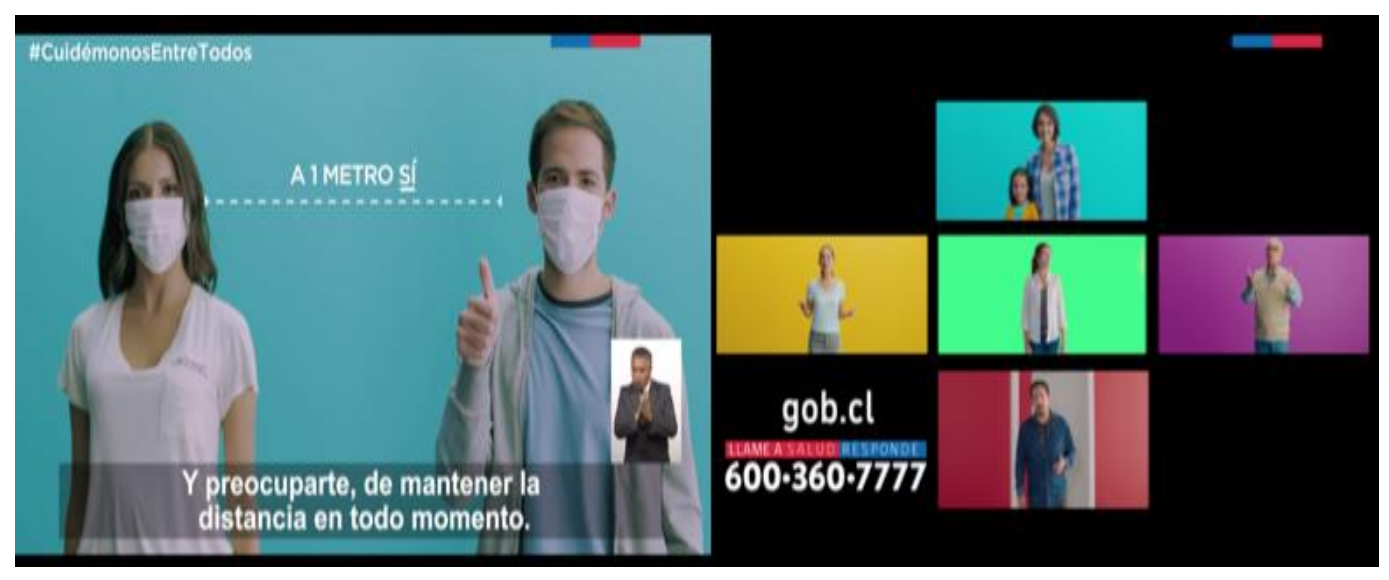

Fuente: MINSAL.

Referente a los discursos escritos y sonoros, las cápsulas revisadas comparten la subcategoría de interés, la que, a pesar de anunciar medidas de prevención, insisten en que la ciudadanía pueda seguir realizando un tipo determinado de acciones que beneficien sus intereses. Y es que en un país neoliberal la economía es algo central para el funcionamiento y bienestar de la nación (La Tercera, 2020a). En este sentido, pese al confinamiento, se insta a la ciudadanía a pagar las cuentas desde sus casas y a salir a trabajar, pero "sólo si es necesario" (28-03-2020_A). De esta manera, los grupos de poder buscan mediar los discursos para cumplir intenciones concretas, desplegando estrategias de consecución (Calsamiglia y Tusón, 2012). 


\section{d. Topoi justicia}

El Gobierno de Chile y el MINSAL, por medio de las cápsulas, lanzan la campaña preventiva con la subcategoría de eslogan mediante la frase "Hazlo por ti y por todos" y el hashtag \#Cuidémosnosentretodos. Ambos mensajes son expuestos en los tres recursos semióticos abordados en esta investigación y son ocupados en una frecuencia de 77 veces, siendo enunciado y reforzado en lo escrito y en lo visual. Esta estrategia tiene el fin de llegar transversalmente a toda la ciudadanía, buscando representar a todos los individuos y, a su vez, hacerlos partícipes de los espacios de prevención y cuidado. Desde la variable de la escritura, al referirse a dar mensajes en primera persona, se compromete un cuidado de parte del gobierno hacia los receptores, pero también se hace un llamado personal al bienestar nacional. En este mismo punto, a nivel visual, este mensaje se expone de forma incongruente ante la realidad nacional, presentando personas que sólo cumplen cánones visuales determinados en desmedro de la diversidad presente en el país. Por tanto, el eslogan que busca construir y justificar un escenario positivo, inclusivo y directo es representado por segmentaciones de presencia -de lo que debe ir y lo que no-, evidenciando un trato preferente que esconde una omisión (Wodak, 2003). En este sentido, se puede decir que la (no)presencia de la diversidad chilena representada en las personas presentes en las cápsulas depende de la perspectiva creativa y las estrategias de quien las crea. Sin embargo, esto también es validado por el organismo nacional, el cual permite exponer un tipo de 'realidad' de acuerdo a lo que quiere mostrar tanto en contenido, como a nivel ideológico y valorativo del entorno (van Dijk, 1999).

Figura 8: Subcategoría posibilidades (28-05-2020_A).

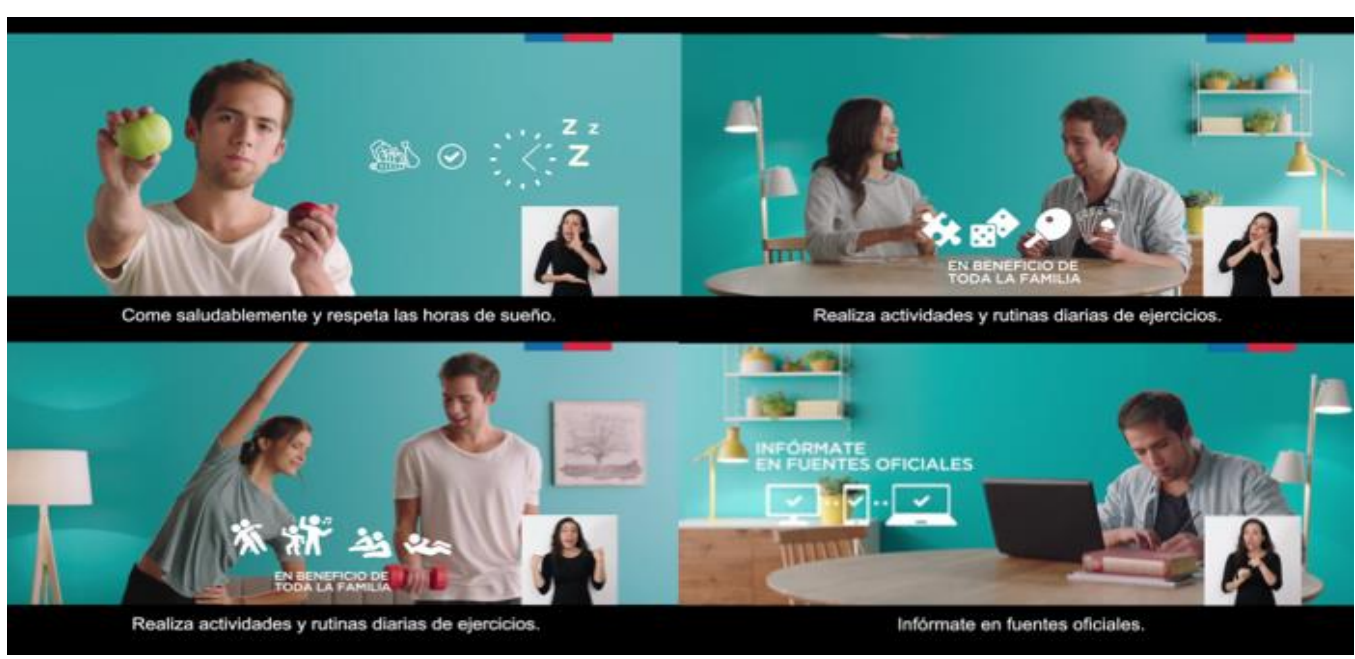

Fuente: MINSAL.

Otra subcategoría argumental fue la de posibilidades (Figura 8), perteneciente al topoi justicia. Esta figura es la encargada de que se ejecuten acciones que aboguen por la igualdad de condiciones; sin embargo, de acuerdo a la realidad país, este mensaje presenta incongruencias 


\section{Javiera Palacios}

igualmente. Por ejemplo, se insta a tener una alimentación saludable (Fig. 8, arriba-izquierda), mientras prevalece la problemática del hambre y las ollas comunes en poblaciones (El Mostrador, 2020c); se invita a la población a realizar actividades de ejercicio (Fig. 8, arriba-derecha; abajoizquierda), mientras muchos viven en espacios reducidos con un grupo familiar amplio (El Mostrador, 2020b); o se recomienda estudiar e informarse (Fig. 8, abajo-derecha), volviendo al topoi utilidad, presuponiendo que todos los chilenos cuentan con acceso a internet o un aparato donde conectarse y realizar estas acciones (Subtel, 2018).

De esta manera, según la observación de los recursos semióticos, se puede exponer que el uso de la subcategoría posibilidades tiene como objetivo orientar las formas de actuar de forma 'ideal', direccionando el quehacer de la población según una visión de la realidad acomodada en favor de aquellas personas que sí pueden acceder a estos espacios, objetos y alimentos -y, por ello, cumplir con todas las recomendaciones de cuidado- en desmedro de otras que no pueden. Como resultado de esto, y de acuerdo a las perspectivas consideradas en esta subcategoría, se establecen cánones socioculturales y rotulaciones económicas capaces de cosificar las identidades nacionales (van Dijk, 1998) en este caso, la chilena.

\section{Conclusiones}

Los discursos preventivos en las cápsulas de la campaña del Ministerio de Salud y el gobierno chileno exponen de forma efectiva informaciones de cuidado a la población, a través de mensajes cortos y reforzados por los recursos semióticos multimodales, los cuales se manifiestan de forma mayoritaria en el topoi utilidad. En el caso de los otros tres topoi analizados -inutilidad, justicia y definición-, se observaron líneas estratégicas de tratamiento discursivo en distintos fotogramas (visual), oraciones (sonoro) y frases (escritas). Se destaca que estas líneas propuestas no son cuestionadas por la población, y es que han sido difundidas por los grupos de poder desde muchas formas y formatos que, en la actualidad, representan informaciones que se dan por sentadas; es decir, que ya son parte del conocimiento general y validadas como tal, tanto a nivel de gráfico, mensaje y forma. De esta manera, por medio del objetivo de investigación, se pudo comprobar que los discursos orientados a la crisis sanitaria y el cuidado del COVID-19 responden a estrategias discursivas explícitas e implícitas incongruentes entre sí. Esto se evidencia en la mayoría de los mensajes analizados, los cuales, si bien fueron enunciados en beneficio transversal a la población, sólo se dirigían -o hacían sentido- a una parte determinada de ella. En definitiva, se aprecian informaciones segmentadas en favor de aquellos grupos sociales que cumplen con los cánones establecidos por la autoridad y que, por su condición, tienen margen de acción más amplio que el resto para resguardar su bienestar y, por ende, ejercer una prevención y cuidado exitoso.

Se puede concluir, por tanto, desde una perspectiva crítica, que los argumentos presentes en estas cápsulas segregan y refuerzan los imaginarios desiguales de la población chilena, imaginarios expresados en una distinción entre aquellos grupos sociales que pueden prevenir el contagio por virus y cuidarse: el nosotros; y quienes deben exponerse para mantener la economía 


\section{Javiera Palacios}

y, por ende, el funcionamiento del país: los otros. En el ámbito de mediación pública, el objetivo de los grupos de poder, por medio de estos cortos informativos, es "educar a la población" (El Mostrador, 2020a; DOS, 2020); sin embargo, el hecho de que estas informaciones no atendieran efectivamente a todas las realidades del público objetivo enunciado evidencia lo contrario. Y es en el carácter implícito donde aparecen, a su vez, dos posibles nuevas lecturas: la primera, en su sentido inmediato, vinculada a una desconexión entre los intereses del gobierno chileno frente a los "otros" intereses de la población común, y que forma parte central de las consignas argüidas en el estallido social chileno; y la segunda, en torno al rol histórico que los grupos de poder desempeñan en favor de sus intereses ideológicos, fomentando la rotulación de cánones y estereotipos de forma estratégica y reforzando, por medio de la multimodalidad de las cápsulas analizadas, diferencias estructurales en una sociedad en crisis.

En este proceso, las estrategias mediadas de los topoi, presentes en los diversos recursos analizados, alimentan las representaciones mentales contextuales e históricas que cosifican la imagen de país ideal, tanto en la forma ideológica-representacional de la población a nivel racial y físico, como en cuanto a intereses y bienestar asociados a privilegios sociales. Estas informaciones son evidentes y sutiles. Transitan sustentadas por una base ideológica sólida y ya cotidiana que, más que generar críticas en la población, circulan libremente validándose en los modelos mentales. De esta forma, se pudo demostrar de forma crítica que los mensajes de cuidado de parte de la autoridad no velan por todos los chilenos, sino que responden sólo a los intereses del nosotros, es decir, del endogrupo que comparte sus ideologías.

Para finalizar, se puede reafirmar que la observación de las cápsulas desde la multimodalidad y la perspectiva de los estudios críticos del discurso, en su conjunto, conforman una herramienta fundamental para develar los sesgos de poder y la segmentación social. De esta manera, a través de los diferentes recursos semióticos, se pueden triangular y entender las elecciones discursivas de los grupos de poder, la disposición de la jerarquía de información y los énfasis utilizados con el fin de reforzar las ideas claves ideológicas. Con esto, se justifica la inclusión o la exclusión para fines específicos (Wodak, 2003), los cuales tienen consecuencias y efectos sociales, cognitivos y materiales (Fairclough, 2003) en la audiencia que los recibe. No obstante, cabe señalar que la sociedad no es una masa acrítica, sino que tiene un rol clave que cumplir: el de interpretar los mensajes de poder y, desde las bases, construir nuevos mensajes, visibilizar las diversidades y dar respuestas. 


\section{Referencias}

Abril, G. (2007). Análisis crítico de los textos visuales. Mirar lo que nos mira. Madrid: Síntesis. Anscombre J. \& Ducrot, O. (1988). La argumentación en la lengua. Madrid: Gredos.

Armada de Chile. (2020, 22 de marzo). Ejército se despliega en apoyo y control de aduanas sanitarias. Ejército de Chile. Recuperado de https://www.ejercito.cl/prensa/visor/ejercitose-despliega-en-apoyo-y-control-de-aduanas-sanitarias

Calsamiglia, H. y Tusón, A. (2012). Las cosas del decir. Manual de análisis del discurso. Barcelona: Ariel.

Castells, M. (2009). Comunicación y poder. Madrid: Alianza.

Chileatiende. (2020, 20 de marzo). Salvoconducto individual. [Información nacional]. https://www.chileatiende.gob.cl/fichas/71060-salvoconducto-individual

Colegio Médico de Chile (COMED) (2020, 15 de marzo). Colegio Médico de Chile solicita a la autoridad extremar medidas para enfrentar pandemia de COVID-19. [Solicitud del gremio].http://www.colegiomedico.cl/colegio-medico-de-chile-solicita-a-la-autoridadextremar-medidas-para-enfrentar-pandemia-de-covid-19/

División de Organizaciones Sociales (DOS) (2020, 16 de abril)._\#CuidémonosEntreTodos ¡Hazlo por ti y por todos! Ministerio Secretaría Nacional de Gobierno - Gobierno de Chile. Recuperado de https://dos.gob.cl/cuidemonosentretodos-hazlo-por-ti-y-por-todos/

Domingo, D. (2020). No era paz, era silencio. El sonido en el paisaje sociosemiótico urbano del "Estallido social" chileno desde los ECDM. Revista Árboles y Rizomas. 2(2), 44-68. https://doi.org./10.35588/ayr.v2i2.4611

El Mostrador. (2020a, 2 de abril). En medio de crisis sanitaria: Subsecretaría de Salud Pública contrató campaña comunicacional a raíz del Coronavirus por $\$ 400$ millones. El Mostrador. Recuperado de https://www.elmostrador.cl/noticias/pais/2020/04/02/en-medio-de-crisissanitaria-subsecretaria-de-salud-publica-contrato-campana-comunicacional-a-raiz-delcoronavirus-por-400-millones/

El Mostrador. (2020b, 7 de agosto). Colmed y la U. de Chile apuntan al "hacinamiento" como el gran riesgo del plan de desconfinamiento del Gobierno. El Mostrador. Recuperado de https://www.elmostrador.cl/destacado/2020/08/07/colmed-y-la-u-de-chile-apuntan-alhacinamiento-como-el-gran-riesgo-del-plan-de-desconfinamiento-del-gobierno/

El Mostrador. (2020c, 22 de mayo). Ollas comunes: respuesta social ante la crisis de hambre que trae el Coronavirus. El Mostrador. Recuperado de https://www.elmostrador.cl/braga/2020/05/22/ollas-comunes-respuesta-social-ante-lacrisis-de-hambre-que-trae-el-coronavirus/

Fairclough, N. (2003). Analysing discourse: Textual analysis for social research. Londres y Nueva York: Routledge.

Fairclough, I. \& Fairclough, N. (2012). Political discourse analysis. Londres y Nueva York: Routdlege.

Ghio, E; Navarro, F. \& Lukin, A. (2017). Nuevas formas de significar: un desafío para la lingüística aplicada. En E. Ghio, F. Navarro, y A. Lukin (Eds.). Obras esenciales de M.A.K. Halliday (pp. 139-181). Santa Fe: Universidad Nacional del Litoral. 
Instituto Nacional de Estadísticas (INE). (2017). Radiografía de género: pueblos originarios en Chile 2017. Recuperado de https://historicoamu.ine.cl/genero/files/estadisticas/pdf/documentos/radiografia-de-genero-pueblosoriginarios-chile2017.pdf

Instituto Nacional de Estadísticas (INE). (2019). Estimación de personas extranjeras residentes habituales en Chile. Recuperado de https://www.extranjeria.gob.cl/media/2020/06/estimaci\%C3\%B3n-poblaci\%C3\%B3nextranjera-en-chile-2019-regiones-y-comunas-metodolog\%C3\%ADa.pdf

Íñguez, L. (2006). Análisis del discurso. Manual para las ciencias sociales. Nueva edición revisada y ampliada. Barcelona: UOC.

Kress, G. \& van Leeuwen, T. (1996). Reading images: The grammar of visual design. Londres y Nueva York: Routledge.

La Tercera (2020a, 7 de abril). José Manuel Silva, de Larrain Vial: "La historia de la industria de las aerolíneas es bastante compleja y, en tiempos normales las dejan quebrar". La Tercera. Recuperado de https://www.latercera.com/pulso-trader/noticia/jose-manuel-silva-delarrainvial-la-historia-de-la-industria-de-las-aerolineas-es-bastante-compleja-y-entiempos-normales-las-dejanquebrar/GMRXI4IDANGP3HZ3ABXFH5AOE4/

La Tercera (2020b, 14 de mayo). Izkia Siches realiza duras críticas a gestión del Minsal tras reunirse con Blumel: "Yo no sé en qué país viven las autoridades de Salud". La Tercera. Recuperado de https://www.latercera.com/nacional/noticia/izkia-siches-realiza-durascriticas-a-gestion-del-minsal-tras-reunirse-con-blumel-yo-no-se-en-que-pais-viven-lasautoridades-de-salud/ZOJIMUJLBRAYHCP5NTTSIQU24Q/

Luzón, M. (1997). Intertextualidad e interpretación del discurso. EPOS, (13), 135-149. https://doi.org/10.5944/epos.13.1997.10013

Meganoticias. (2020, 30 de junio). Tratamiento contra el coronavirus: ¿Cuál sería su precio en Chile? Meganoticias. Recuperado de https://www.meganoticias.cl/calidad-devida/306246-precio-remdesivir-tratamiento-coronavirus-390-dolares-coronavirusppx03.html

Ministerio de Salud de Chile (MINSAL). (2020a, 3 de marzo). Ministerio de Salud confirma primer caso de coronavirus en Chile. Recuperado de https://www.minsal.cl/ministerio-desalud-confirma-primer-caso-de-coronavirus-en-chile/

Ministerio de Salud de Chile (MINSAL). (2020b, 16 de julio). Autoridades de salud destacan disminución de casos nuevos en la Región Metropolitana. Recuperado de https://www.minsal.cl/autoridades-de-salud-destacan-disminucion-de-casos-nuevos-enla-region-metropolitana/

Navarro, F. \& Tromben, C. (2019). "Estamos en guerra contra un enemigo poderoso, implacable": los discursos de Sebastián Piñera y la revuelta popular en Chile. Literatura y Lingüística, 40, 295-324. https://doi.org/10.29344/0717621x.40.2083

O’Halloran, K. (2012). Análisis del discurso multimodal. ALED, 12(1), 75-97.

Pardo, N. (2007). Cómo hacer ACD. Una perspectiva latinoamericana. Bogotá: Frasis.

Richardson, J. (2004). (Mis)Representing Islam: the racism and rhetoric of British Broadsheet newspapers. Ámsterdam y Filadelfia: Benjamins. 


\section{Javiera Palacios}

Ruiz, E. \& Estrevel, L. (2008). La ideología y la transformación del sujeto. Universitas Psychologica, 7(1), 33-41.

Sáez, J. (2014). Racismo en la prensa chilena: deconstrucción de la frontera simbólica en la representación social del mapuche como el otro. Contextos : Estudios de Humanidades y Ciencias Sociales, (32), 121-134.

Subsecretaría de Telecomunicaciones de Chile (SUBTEL) (2018, 17 de mayo). Novena Encuesta $\begin{array}{llllll}\text { Accesos } & \mathrm{y} \text { Usos } & \text { de }\end{array}$ https://www.subtel.gob.cl/wpcontent/uploads/2018/05/ppt_usos_may2018.pdf

Taboada, M. (2018). Abordaje de sujetos migrantes y procesos migratorios en libros de texto de Ciencias Sociales. Un análisis de caso. Educación, 42(1), 2-19. https://doi.org/10.15517/revedu.v42i1.23187

van Dijk, T. (1997). Racismo y análisis crítico de los medios. Barcelona: Paidós.

van Dijk, T. (1998). Texto y contexto. Madrid: Cátedra.

van Dijk, T. (1999). El análisis crítico del discurso. Barcelona: Anthropos.

van Dijk, T. (2003). Ideología y discurso. Barcelona: Ariel.

van Dijk, T. (2005a). Politics, ideology and discourse. En R. Wodak (Ed.), Elsevier Encyclopedia of Language and Linguistics (pp. 728-740). Ámsterdam: Elsevier.

van Dijk, T. (2005b). Ideología y análisis del discurso. Utopía y Praxis Latinoamericana, 10(29), $9-36$.

van Dijk, T. (2012). Discurso y Contexto. Un enfoque sociocognitivo. Barcelona: Gedisa.

van Leeuwen, T. (1999). Speech, music, sound. Londres: Macmillan.

Wodak, R. (2003). El enfoque histórico del discurso. En R. Wodak y M. Meyer. (Eds.) Métodos de análisis crítico del discurso (pp. 101-142). Barcelona: Gedisa.

Wodak, R. (2009). The discourse of politics in action: Politics as usual. Londres: Palgrave Macmillan.

Wodak, R. (2011). La historia en construcción / La construcción de la historia. La "Wehrmacht alemana" en los recuerdos colectivos e individuales de Austria. Discurso \& Sociedad, 5(1), 160-195.

Wodak, R. (2015). The politics offear. What rightwing populist discourses mean. Londres: SAGE. Wodak, R. \& Meyer, M. (2015). Métodos de análisis crítico del discurso. Barcelona: Gedisa.

Žagar, I. (2010). Topoi in critical discourse analysis. Lodz Papers in Pragmatics,6(1), 3-27. https://doi.org/10.2478/v10016-010-0002-1 